\section{Wireless Data Acquisition and Control Systems for Agricultural Water Management Projects}

\author{
S. Shukla, C.Y. Yu, J.D. Hardin, and F.H. Jaber
}

AdDitional INDEX wORDs. remote datalogging, wireless dataloggers, real-time data monitoring, remote control, spread spectrum

SuMmARY. Continuous monitoring of hydraulic/hydrologic data for managing water for horticultural crops has been a challenge due to factors such as data loss, intensive resource requirements, and complicated setup and operation. The use of state-of-the-art wireless spread spectrum communication technology and wireless data acquisition and control (WDAC) systems for agricultural water management is discussed in this paper. The WDAC technology was applied to a research project where lysimeters were used for water quantity and quality studies for vegetables. Two types of WDAC networks, master-slave and peer-to-peer WDAC networks, are discussed. The WDAC system linked the wireless dataloggers to a network to make real-time data available over the Internet. The use of WDAC made it possible to collect real-time data and control the experiment (e.g., frequency of data collection) remotely through the Internet. The WDAC system for the lysimeter study was compared to a commonly used manual system with regard to potential instrument damage, data loss, ease of data collection and analyses, and total cost of monitoring. The advantages of the WDAC include: reduced equipment losses from natural disasters (e.g., lightning), improved equipment maintenance, reduced data loss from faulty equipment, higher project personnel efficiency, and real-time involvement by a dispersed team. The total cost of the WDAC system $(\$ 65,750)$ was about half that of the manual system $(\$ 130,380)$. The WDAC system was found to be an effective tool for agricultural water management projects.

$\mathrm{E}$ ffective hydraulic/hydrologic monitoring studies are critical for agricultural water resources management programs (Glasgow etal., 2004). Experimental studies often require continuous monitoring of several parameters, such as flow depth (stage), discharge, and soil moisture at one or more sites (e.g., multiple sites within an agricultural field or multiple fields) in remote locations. The collection of continuous hydraulic/hydrologic data from multiple sites that may be distant from the research institution presents formidable challenges to the researcher with regard to travel time involved in data retrieval, personnel, data recording and georeferencing errors, and financial resources (Glasgow et al., 2004; Lundquist et al., 2003; Teillet et al., 2002).

To monitor changes in various

Acknowledgment. The lysimeter project was funded through the Southwest Florida Water Management District and the South Florida Water Management District. Thanks are also due to Mr. Saurabh Srivastava, graduate research assistant, for his help. The use of the Campbell Scientific, Inc., Sentek Pty. Ltd., and other companies' instruments were for example purposes only and do not reflect endorsement from the University of Florida. hydraulic/hydrologic parameters in real-time on a personal computer (PC), the datalogging instrument and PC must be linked together. Typically, the standard RS-232 serial bus associated with the computer communication port has been used for the communication link. However, this method poses a significant limitation, since a RS-232 serial connection links only one datalogger with one computer (Cheek and Wilkes, 1994). For experiments in which several sites are monitored through individual dataloggers, it is not possible to access the data at each site through a single computer without complex serial multiplexing techniques. Serial multiplexing also has its limitations because multiplexing requires that sites be located close (e.g., within $50 \mathrm{ft}$ ) to each other. It is impractical to use multiplexing for sites distantly located from each other (e.g., 10 miles) due to the high likelihood of physical and/or electrical damage to the wires and dataloggers.

If the software application associated with the datalogger allows data to be examined only after the download, errors or malfunctions cannot be detected when they occur. Furthermore, the manual retrieval method is costly and difficult when monitoring multiple sites. The cost of monitoring becomes increasingly expensive as the number of processes and/or number of sites to be monitored increases. The main factors increasing costs are personnel and travel costs associated with manual download.

In addition to the operational difficulties in multi-site monitoring, the damage to instruments by natural disasters, farm machinery, wildlife or livestock, or human interference can also pose a major challenge and results in loss of data. The damage to instruments is of special concern in areas prone to frequent lightning. Although lightning protection devices are available, in most cases they are not able to protect field instrumentation from a direct strike. In areas where lightning frequency is high, long wire runs should be minimized to limit damage to hard-wired data networks. The potential for damage is especially high in southern Florida, which has the highest mean annual cloud-to-ground lightning flash densities in the U.S. (Orville and Huffines, 2001).

The use of the latest WDAC technology offers the researcher a viable solution to problems of: 1) real-time multi-site data access, 2) data acquisition on an as-needed basis, 3 ) prevention of instrument damage due to natural and other incidents, 4) complex site installation including intricate wiring connections, and 5) costly and time-consuming travel to access the data.

This paper discusses the essential components of the currently available WDAC systems suitable for horticul-

\begin{tabular}{llll}
\hline $\begin{array}{l}\text { Units } \\
\begin{array}{l}\text { To convert U.S. to SI, } \\
\text { multiply by }\end{array}\end{array}$ & U.S. unit & SI unit & $\begin{array}{l}\text { To convert SI to U.S., } \\
\text { multiply by }\end{array}$ \\
\hline 0.4047 & acre(s) & ha & 2.4711 \\
0.3048 & $\mathrm{ft}$ & $\mathrm{m}$ & 3.2808 \\
2.54 & inch(es) & $\mathrm{cm}$ & 0.3937 \\
25.4 & inch(es) & $\mathrm{mm}$ & 0.0394 \\
1.6093 & mile(s) & $\mathrm{km}^{2}$ & 0.6214 \\
2.5900 & mile & $\mathrm{km}^{2}$ & 0.3861
\end{tabular}


tural studies and the application of such systems in real-time hydrologic monitoring in remote locations.

Specific objectives are to:

1) Review the WDAC applications in water resources and discuss the essential components, integration, and use of WDAC systems for remote, multi-site water management projects.

2) Design the WDAC system from commercially available components and apply it to an example project on evapotranspiration from horticultural crops and compare the WDAC system to the traditional system.

\section{Development and use of WDAC technology}

In 1985, the Federal Communications Commission (FCC) allocated three frequency bands for a radio transmission technology, known as spread spectrum communication technology (SSCT). The goal of this allocation was to encourage usage toward local data communication. Although spread spectrum product users do not require FCC certified license, the manufacturers must meet FCC spread spectrum regulations. The SSCT, originally developed by the U.S. Department of Defense, works as follows: the transmitter takes the input data and spreads it through a predefined method. The receiver understands the predefined method and reassembles the signal so that the data can be interpreted. The advantages of using the wireless spread spectrum technology are: 1) free FCC site licenses to the end users; 2 ) great immunity to interference and noise; and 3) dynamic multi-channel changes through software using either direct-sequence or frequency-hopping techniques.

There have been several reported uses of WDAC in water resources projects that resulted in ease of data collection from remote and difficult to reach sites and/or significant savings in operating costs (Cheek and Wilkes, 1994; Cockerham and Ortega, 1989; Damas et al., 2001; Lundquist et al., 2003; Maes and Steffey, 1996; Sauter and McDonnell, 1994). Most of these WDAC systems were only monitoring a single parameter such as flow or $\mathrm{pH}$. Furthermore, these systems were custom built for unique applications, which makes them costly to use for more generalized monitoring projects. In most cases, the system development costs could be a major part of a con- strained research budget in agricultural water management studies. Because of these issues, it was not normally possible to apply these systems to financially constrained water quantity and quality research projects.

The Sensor Web, developed by NASA (Delin et al., 2005), is a system comprised of several spatially distributed sensors. It is an emerging new technology that uses wireless transmission for data collection. The Sensor Web performs similar tasks that a WDAC system can do, however this technology is not yet commercially available and thus would require considerable efforts to customize it for a specific application.

Recent advances in data acquisition and control have made it possible to use an integrated system of dataloggers and wireless communication for horticultural projects. Examples of off-the-shelf systems include the CR205 [Campbell Scientific, Inc. (CSI), Logan, Utah (2003)] and SMS85 [EDAC Electronics Australia $\mathrm{P} / \mathrm{L}$, Boronia, Australia (Reed Business Information, 2003)]. With such systems, the user can directly check and report the working condition of field equipment at remote sites, wirelessly retrieve the data, and remotely control a device through a variety of communication systems such as standard telephone line, mobile phone, global system for mobile (GSM) or cellular digital packet data (CDPD) network, or computer. For example, substantial water and cost savings can be achieved if one could control irrigation based on real-time soil moisture data. These systems usually have a built-in security system to avoid unauthorized access. To demonstrate the use of wireless technology for horticultural projects, we selected and tested the CR205 datalogger by CSI.

The CR205 can measure, process, store, and transmit the data from compatible sensors via its on-board spread spectrum transceiver. It communicates with other dataloggers through: 1) wireless communication using the PakBus protocol, a simplified variation of Internet protocol (IP); and 2) direct communication using the serial communication (RS-232) port. The CR205 datalogger (Fig. 1) has five single-ended (SE) analog input ports, two pulse input ports, two digital control input/output $(\mathrm{I} / \mathrm{O})$ ports $(\mathrm{Cl}$ and C2), two excitation channels $(2.5$ or
$5 \mathrm{~V}$ ), and one switched battery port. One of the digital control ports can also be configured to connect the addressable SDI-12 (serial digital interface at 1200 -baud) interface sensors.

The SDI-12 interface was developed by a group of water instrumentation users [FTS Forest Technology Ltd., Blaine, Wash. (2000)]. The SDI12 digital protocol is a set of rules that define how a sensor communicates with a datalogger. Circuitry and intelligence are added to create a "smart" device that allows the user and the manufacturer to program and calibrate the sensor independent of the datalogger as opposed to analog voltage or current sensor outputs where device calibration and data collection are carried out by a user program incorporated in the datalogger. The SDI-12 digital signal requires low power and is resistant to data errors. Compared to SDI-12 sensors where the sensor's analog signal is converted to digital at the sensor itself, an analog signal is more likely to be corrupted due to interference from a variety of sources (e.g., electromagnetic fields, radio signals, lightning, and cable lengths) (FTS Forest Technology Ltd., 2000). The SDI-12 interface can be used to communicate with "smart" sensors that have local microprocessors to handle data communications, sensor calibration, and the SDI-12 communication protocol. Many sensors used in water resources applications have the SDI-12 interface. Examples include soil moisture, conductivity, dissolved oxygen, water level, $\mathrm{pH}$, and temperature. Not all water-monitoring devices are SDI-12 compatible (e.g., irrigation flow meters).

The unique advantages of the SDI-12 interface compared to analog sensors includes: 1) the ability to use long cables of varying length from the sensor to the datalogger such that the cable length is governed more by the voltage drop allowed by the remote microprocessor than by any other consideration: 2 ) the ability to transmit data from several sensors via one cable (e.g., multiple soil moisture data from several sensors at different locations and/or multiple depths) versus one sensor per cable required for voltage/ current sensors; 3 ) simplifying the sensor replacement/servicing with ability to plug/unplug sensors instantly from the datalogger system without affecting other components in the system; 4) the ability to replace faulty sensors 


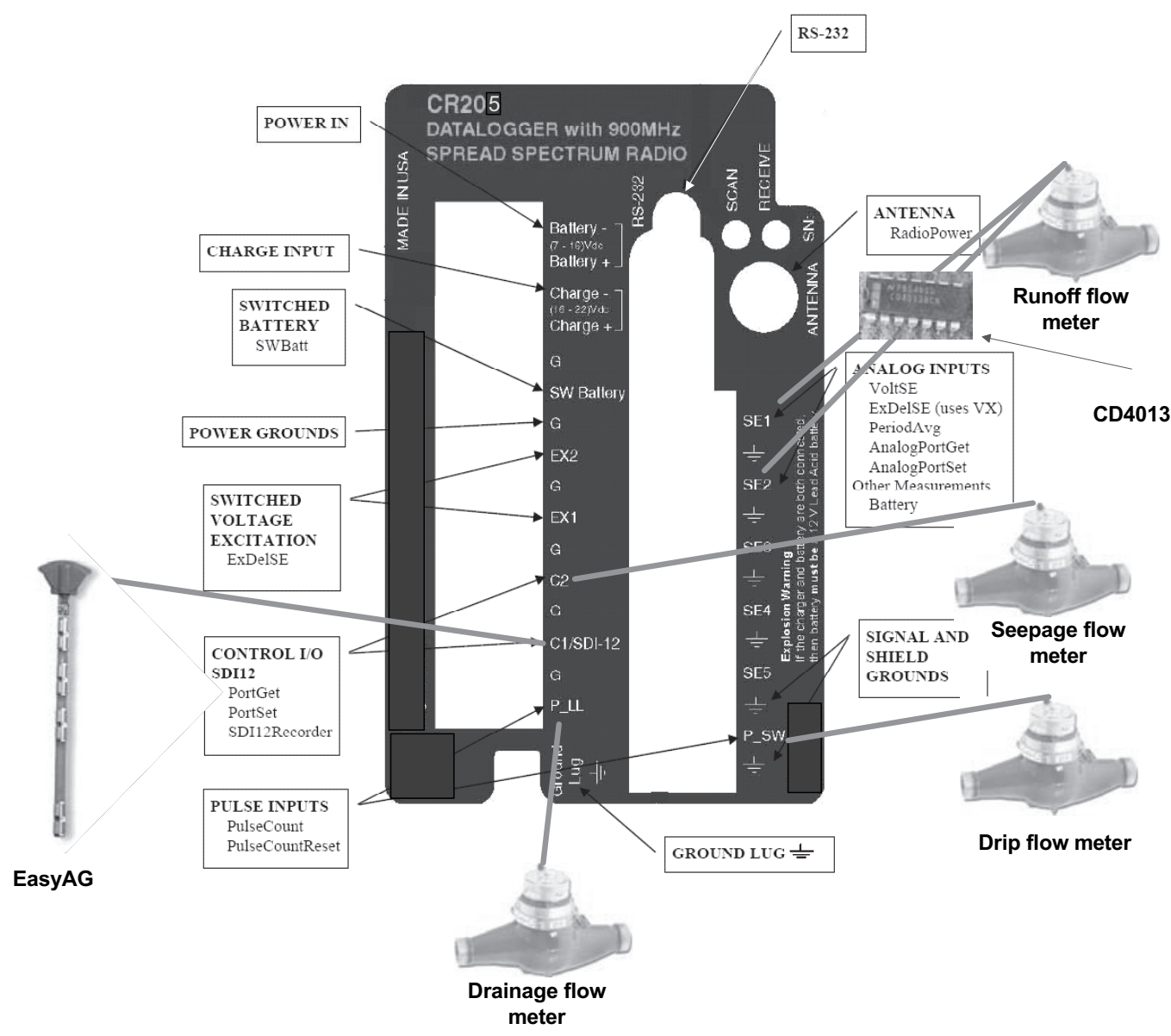

Fig. 1. Schematic for soil moisture and flow measurements using a CR205 datalogger (Campbell Scientific, Inc., Logan, Utah); RS-232 = data port using recommended standard 232; SDI12 = serial digital interface at 1200-baud; CD4013 = dual D-type flip-flop integrated circuit chip; EasyAG = EasyAG soil moisture probe (Sentek Pty Ltd, Stepney, Australia).

without modifying the datalogger program (calibration information is stored within the sensor); and 5 ) the ability to access an increasing number of sensors and dataloggers without using sensor specific protocols and custom wiring and programming.

\section{WDAC design and example application for agricultural water management projects}

A WDAC system was designed to monitor multiple sites within a small area close to the research facility. The WDAC system is currently being used for an evapotranspiration and water quality study at the University of Florida (UF), Institute of Food and Agricultural Sciences (IFAS), Southwest Florida Research and Education Center, Immokalee, Fla. The study involves quantifying the crop coefficient for vegetable crops [e.g., watermelon (Citrullus lanatus)] for the development of irrigation best management practices (BMPs) (Shukla et al., 2006).
The multiple-lysimeter project was started in 2003 to quantify the evapotranspiration (ET) data for row crops for drip and seepage irrigation systems. A total of six drainage lysimeters $(16 \mathrm{ft}$ long $\times 12 \mathrm{ft}$ wide $\times 4.5 \mathrm{ft}$ deep) were installed in a 1.5 -acre field. In each lysimeter, irrigation, drainage, runoff, and soil moisture data are collected to quantify the ET for vegetable crops. ET is computed as the difference between all water input (irrigation, rainfall) and output (runoff and drainage).

Weather parameters, including rainfall, are collected at a Florida $\mathrm{Au}^{-}$ tomated Weather Network (FAWN) wireless weather station located 150 ft from the lysimeter field. Weather monitoring was not integrated into the wireless system. However, a wireless weather station could be easily integrated with the wireless datalogging system.

INFLOW AND OUTFLOW CONTROL AND MEASUREMENTS. Each of the six lysimeters was instrumented with a number of measurement devices to measure water inflows and outflows, water-table depths, and soil moisture content. The irrigation system was designed to provide water to the lysimeters as well as the surrounding field. The irrigation in the field was scheduled based on soil moisture readings, to maintain the optimum soil moisture.

Figure 2 depicts the field instrument setup used for the lysimeters. The instruments for each of the lysimeters consist of one Sentek EasyAG SDI-12 soil moisture probe [Sentek Pty Ltd, Stepney, Australia (2002)], four flow meters (non-SDI- 12 compatible; pulse output), and one CR205 datalogger. A list of the instruments used for the lysimeter project is given in Table 1 . The Sentek EasyAG soil moisture probe consists of four soil moisture sensors at depths of $10,20,30$, and $50 \mathrm{~cm}$ and an addressable SDI- 12 interface. The built-in SDI-12 interface is programmed to convert the sensor 


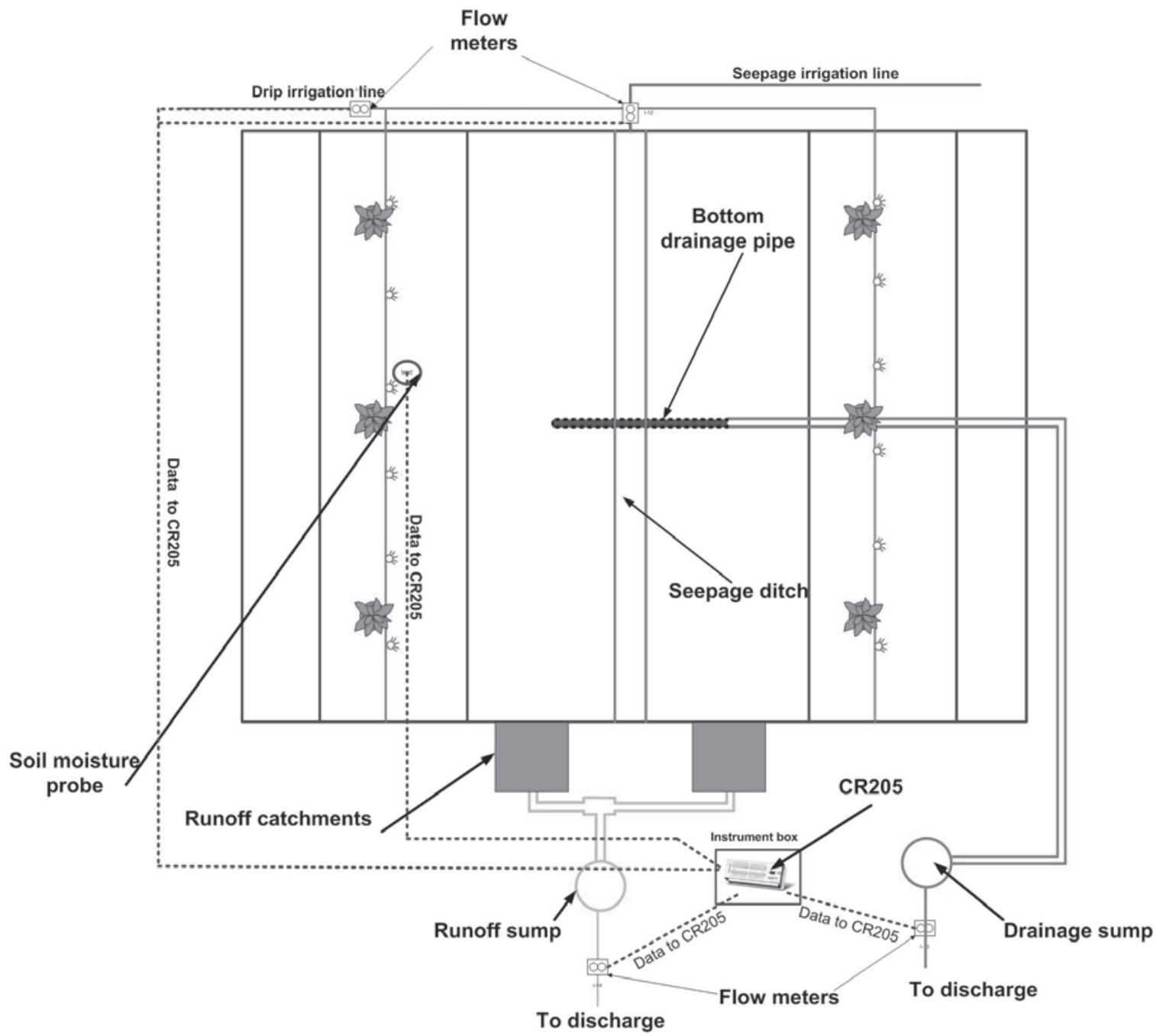

Fig. 2. Field instruments setup for wireless data acquisition and control (WDAC) for a lysimeter (top view) used for evapotranspiration studies. CR205 = CR205 datalogger (Campbell Scientific, Inc., Logan, Utah).

Table 1. Instruments used as parts of the wireless data acquisition and control system (WDAC) installed to monitor six lysimeters used for evapotranspiration water quality studies in Immokalee, Fla.

\begin{tabular}{lll}
\hline Instrument & Model & \multicolumn{1}{c}{ Manufacturer } \\
\hline Wireless datalogger & CR205 & Campbell Scientific, Inc., Logan, Utah \\
Datalogger & CR10x & Campbell Scientific, Inc. \\
Spread spectrum radio & RF 400 & Campbell Scientific, Inc. \\
Soil moisture measurement device & SDI12 Sentek EasyAG Probe & Sentek Pty Ltd., Stepney, Australia \\
Network link interface & NL100 & Campbell Scientific, Inc. \\
Flow meter & $5 / 8 \times 3 / 4$ inch $^{z}$ DLJ & Daniel L. Jerman Co., Hackensack, N.J. \\
Yagi antenna & $6 \mathrm{dBd} \mathrm{Y8066}$ & Antenex Inc., Glendale Heights, Ill. \\
\hline
\end{tabular}

${ }^{2} 1$ inch $=2.54 \mathrm{~cm}$.

data into volumetric water content (percent).

Flow meters were connected to drip (four lysimeters) and seepage (two lysimeters) irrigation lines at the lysimeters to quantify irrigation volume. The drainage from the lysimeters was brought to an external drainage sump from where it was discharged through a pump (Shukla et al., 2006).
The drainage pump was triggered by a water sensor and an electric switch.

The drainage was measured through a flow meter with a pulse output and is connected to a CR205. A similar collection and measurement system was designed for measuring runoff.

DESIGN CONSIDERATION AND USE OF THE WDAC. Human resources and potential for natural disaster (e.g., lightning) were the two driving forces for the design and use of WDAC for the lysimeter project. The study area is located in a high rainfall area (average annual rainfall $=128 \mathrm{~cm} /$ year) (Southeast Regional Climate Center, 2005) and consists of sandy soils with high hydraulic conductivity. High rainfall combined with rapid movement of water and nutrient in the soil required 
a real-time monitoring (every $15 \mathrm{~min}$ ) system to adjust the irrigation management and monitor proper drainage to avoid flooding. A full-time person to manage irrigation was unavailable for the project.

In a prior vegetable water management study, lightning seriously damaged four soil moisture probes and the datalogger, resulting in approximately $\$ 10,000$ of damage to the instruments (Shukla and Pandey, 2003). The study involved five soil moisture probes (with four sensors per probe) that were hard wired to a datalogger. The lightning resulted in plant damage within a circular area of about 20 -ft radius and damaged all the moisture sensors inside as well as outside this circle since they were connected to each other. Because the sensors and the datalogger had to be replaced, data collection was interrupted for almost 1 month, which resulted in loss of data. Use of wireless dataloggers (e.g., CR205) would have resulted in fewer soil moisture probes being lost due to lightning since the four probes would have not been connected to each other. These considerations caused the authors to explore the use of automated instrumentation with wireless radio networks for the lysimeter project. The effect of lightning strikes on a data logging projects is further detailed in the cost comparison section.

Hardware. The WDAC network designed for the multiple-lysimeter project consists of one CRIOX datalogger with RF400 radio frequency modem, six CR205 dataloggers with on-board $915 \mathrm{MHz}$ spread spectrum radios, one Network Link 100 [NL100 (Campbell Scientific, Inc., 2002a)] with a RF400 radio frequency modem as a wireless network interface to the local area network (LAN), and one PC with one RF400 radio modem. The CRIOX datalogger is a measurement and control system with 12 single ended analog channels, two pulse input channels, and eight control/digital ports and can store up to 62,000 data points (upgradable to 1 million data points) in its non-volatile memory (Campbell Scientific, Inc., 2004).

The WDAC network for the lysimeter project uses the RF400 radio frequency modem at the $\mathrm{PC}$ and the on-board radio of the CR2 05 datalogger for communication. The RF400 radio frequency modem acts as a 900 $\mathrm{MHz}$, frequency-hopping, spread spectrum data radio as well as modem. It is designed for point-to-point (a PC to a CRI0X or to a CR205) and point-tomultipoint (PC to CRI0X and/or each CR205) communications. The gain energy of the radio's antennas and the line-of-sight paths between radios affect the communication distance $(0.25$ to 10 miles) to which the data can be wirelessly transmitted. A thick stand of trees can limit the communication distance to $800 \mathrm{ft}$ (Campbell Scientific, Inc., 2005). The line-of-sight transmission range, the distance between the wireless network interface (PC) and the CR205, in this application is approximately $500 \mathrm{~m}$. Both the RF400 and the on-board radios on CR205 use the PakBus packet switching network protocol to transmit information among the CRIOX datalogger, the CR205 dataloggers, the NL100, and the PC. The power source for the CR205s and sensors was a $12-\mathrm{V}$ lead acid marine (gel type) battery that was charged with photovoltaic cells (solar panel) installed on top of the CR205 housing. The marine battery is completely sealed, leak free, and long lasting (batteries installed in 2003 were still operational in 2006).

Figures 1 and 2 depict the field instrument connections with the CR205 datalogger. Three of the four irrigation pulse-output flow meters were connected through one control I/O port, which can act as a pulseinput port, and two pulse-input ports at the CR205 dataloggers. To collect runoff data from the fourth pulseoutput flowmeter, a flip-flop electronic circuit using a common CD4013 dual D-type flip-flop integrated circuit chip (IC) was designed and fabricated to measure the flow using two of the single ended (SE) I/O ports, labeled analog inputs on Fig. 1. The CD4013 is configured as a pulse-input counter. Its output port, is connected to one of the five SE ports on CR2 05 configured as digital input port, while its Reset2 port is connected to another SE port configured as digital output port of CR205 to count the pulses from the runoff flow meter. When the CD4013 receives a pulse from the flow meter, it sends a digital signal to the SEI port to set the port to high status to indicate the arrival of the pulse. The high status is stored and counted by the program within the CR205. Once the high status of the SEI port is counted, the SE2 port is toggled to high status to reset the CD4013.
The NL100, a network link interface, is a device used to facilitate communications between either the CR10X or the CR205 dataloggers and a PC through its Ethernet 10 Base- $\mathrm{T}$ communication link. The NL100 contains a I/O port and a RS-232 port and is connected to the UF-IFAS network. The RS-232 port in the NL100 is configured as a PakBus serial port and connected to a RF400 radio frequency modem to wirelessly transfer the data from the datalogging network to the PC.

Software. Software used for measurement, data collection, and wireless transmission include: 1) Intelligent Probe Configuration Utility (IPCU) (Sentek Pty Ltd., 2002) for the soil moisture measurement device; 2 ) HyperTerminal [Microsoft, Redmond, Wash. (1999)], a program embedded in the Microsoft Windows operating system, for the configurations of the network interface device (NLI00) and radio frequency modem (RF400); 3) PakCom Wireless Communication [PWC (Campbell Scientific, Inc., 2002b)] for the configuration of the CR205 on-board radio; and 4) LoggerNet 2.la (Campbell Scientific Inc, $2002 \mathrm{~b}$ ) for the control of the WDAC devices. LoggerNet and PWC are provided with the datalogger.

The IPCU software is used to configure the Sentek EasyAG SDI12 probe interface (Sentek Pty Ltd., 2002 ) and is provided by the manufacturer. There are four items that must be configured: soil-depth locations, normalization values (air and water counts for zero and 100\% moisture content), coefficients of the calibration equation for each sensor in the probe, and the SDI-12 address of the probe. The configured information is stored in the non-volatile memory of the Sentek EasyAG SDI-12 probe and is used to generate the volumetric water content (VWC) (millimeters $/ 10 \mathrm{~cm}$ ) data from every sensor on individual EasyAG probes. The VWC data are stored in the CR205.

The HyperTerminal software was used to configure the NLI00 to work as a PakBus serial server in a standard TCP/IP network and as a PakBus node in a PakBus network (Campbell Scientific, Inc., 2002a). HyperTerminal is also used to configure the RF400 radio modem for wireless communication.

The PWC software is the control and configuration software for the 


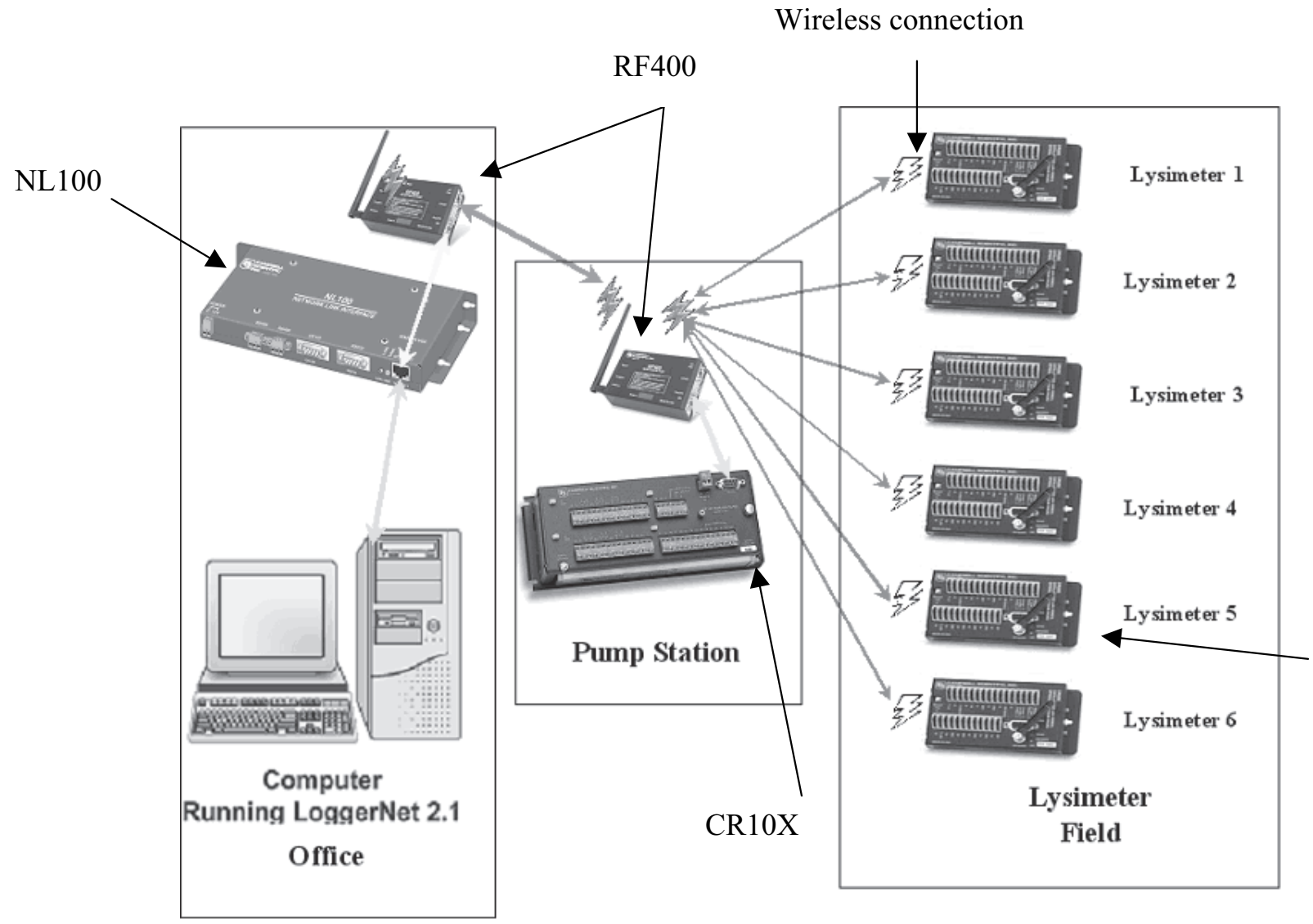

CR205

Fig. 3. Master-slave wireless data acquisition and control system (WDAC) network. The links within the two left blocks are physical, while the interblock connections are wireless. CR205 and CR10X = CR205 and CR10X dataloggers (Campbell Scientific, Inc., Logan Utah); NL100 = Network Link 100 (Campbell Scientific, Inc.); RF400 = RF400 spread spectrum radio (Campbell Scientific, Inc.); LoggerNet 2.1 = datalogger support software (Campbell Scientific, Inc.).

CR205 datalogger (Campbell Scientific Inc., 2002b). The PWC software is used to configure the on-board radio of the CR205. Both the RF400 radio modem and the on-board radio of the CR205 are set at the same radio address, radio net address, radio hop sequence, and standby power mode to enable the wireless data transmissions from the WDAC network to the PC through the NL100.

The PWC could only be used to control and configure the CR205. Therefore, LoggerNet 2.1a, which serves as a control software for the WDAC network was used to control and configure the other datalogger (CRI0X), radio modems (RF400), and the network interface device (NL100). The functions of LoggerNet in the WDAC network include: 1) setup, connect, and monitor the instruments; 2 ) create or edit control programs for the instruments; 3 ) report, view, and monitor the data in real time; and 4) manage and collect data from the dataloggers (Campbell Scientific,
Inc., 2002b). The program language used for the CRIOX is Edlog 32 for Windows. The program language for CR205 is CRBasic Editor, version 1.5.0.7 (Campbell Scientific, Inc., 2002b). Both Edlog 32 and CRBasic programs were written using the editor in LoggerNet to: 1) trigger the Sentek EasyAG SDI- 12 probe to measure the soil moisture content data; 2) count and record the digital pulses from drip, seepage, drainage, and runoff flow meters; 3 ) store all the table-based data; and 4) wirelessly transmit the data from each of the six CR205 to the CR10X and then to the PC in the office. The above-mentioned setup can be implemented by a technician with electronics background within a month. For cases when in-house technical help is not available, the manufacturing company can be contacted to help set up the monitoring system.

Networking. Two types of hardware setups can establish the WDAC network-to-user-interconnectivity. These two types, set up using Log-
gerNet, are: 1) the master-slave and 2 ) the peer-to-peer communication networks.

Figure 3 depicts the configuration of the master-slave WDAC network in LoggerNet for the lysimeter project. The CRI0X with RF400 radio plays the role of master in the WDAC network, and the six CR205s with on-board radios act as the slaves. Each of the six CR205s communicates with the PC via the CRIOX. The CRIOX and each of the six CR205 dataloggers in the WDAC network require a unique PakBus address to transmit the data to the PC. Once data are transmitted to the PC, data are stored in a directory on the hard drive with a "redundant array of inexpensive disks" (RAID) system for backup. Given the known soil field capacity and permanent wilting points at each lysimeter, LoggerNet can report, view, and monitor the data in real time for daily scheduling of irrigation.

Another type of WDAC network evaluated is the peer-to-peer com- 


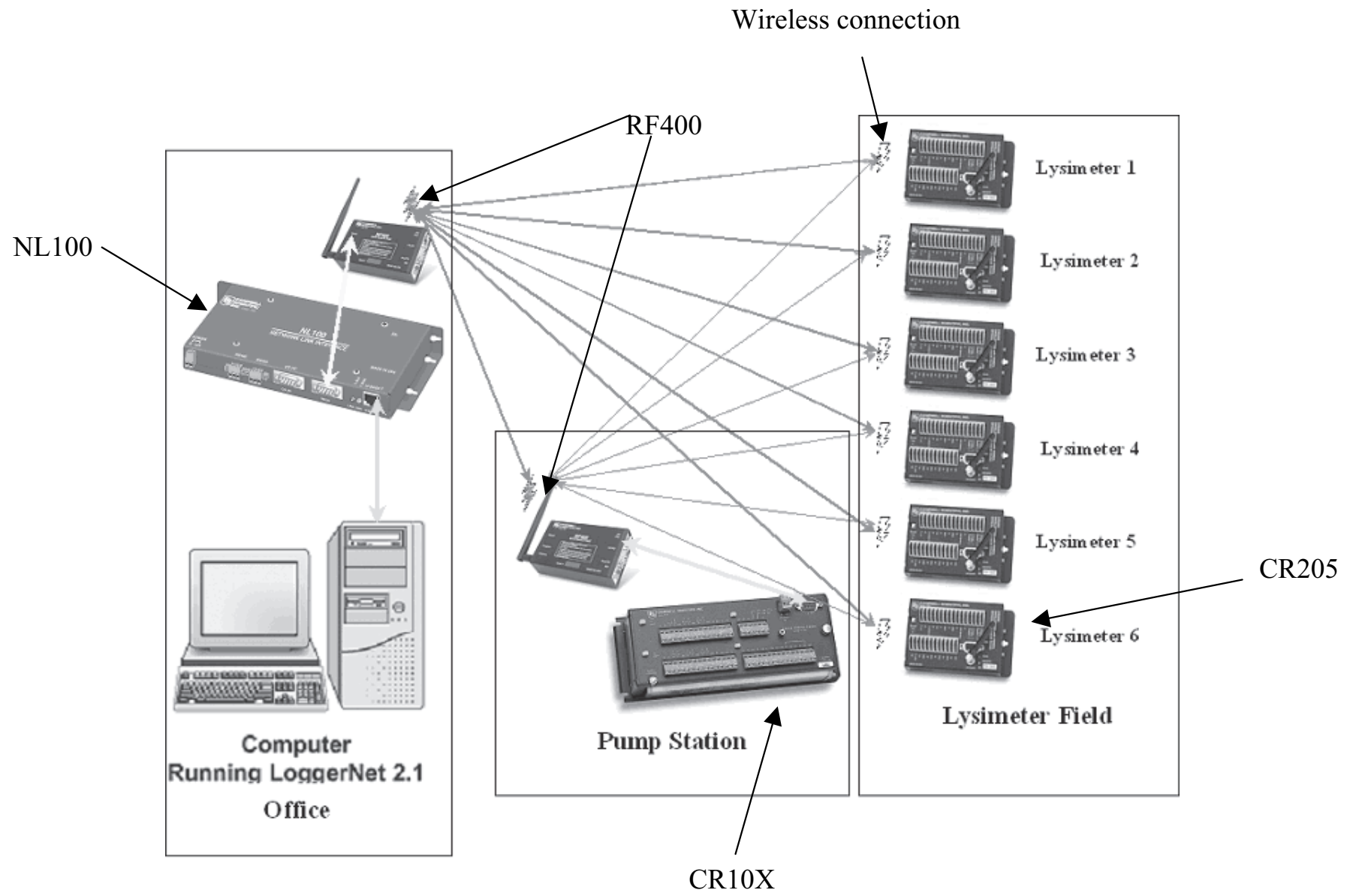

Fig. 4. Peer-to-peer wireless data acquisition and control system (WDAC) network. The links within the two left blocks are physical, while the interblock connections are wireless. CR205 and CR10X = CR205 and CR10X dataloggers (Campbell Scientific, Inc., Logan Utah); NL100 = Network Link 100 (Campbell Scientific, Inc.); RF400 = RF400 spread spectrum radio (Campbell Scientific, Inc.); LoggerNet 2.1 = datalogger support software (Campbell Scientific, Inc.).

munication network. Figure 4 depicts the configuration of a peer-to-peer WDAC network in LoggerNet for the lysimeter project. Both the CRI0X and the six CR205s communicate directly with the PC via the wireless network interface. As in the master-slave setup, the CR10X and each of the six CR205 dataloggers in the WDAC network require a unique PakBus address to transmit data to the PC. The peer-topeer WDAC network is useful where the distance between the $\mathrm{PC}$, the CRIOX, and all CR205s is short (approximately $500 \mathrm{~m}$ ).

Although any of the two communication network could be used for the lysimeter project, depending on the monitoring parameters and research settings, peer-to-peer communication may have advantages over the master-slave network and vice versa. The advantages of the peer-to-peer system are: 1) communication of data from one peer (e.g., CR205 at lysimeter 1) to another peer (e.g., CR205 at lysimeter 2 ) for controlling a device such as an irrigation pump; 2) improved data transmission redundancy due to using multiple paths for collecting the data at the base station PC (in case one of the sites is off line, another peer station can serve as a repeater in the network); 3 ) ability to transfer data from a peer station to another peer station directly; and 4) simpler implementation of a network since the data transmission path is selected by the network, not the user. For example, soil moisture data from one of the lysimeters could be used to control irrigation in another lysimeter that does not have the SDI12 soil moisture probe due to cost considerations. It should be noted that while CR205 (e.g., lysimeter 1) to CR205 (e.g., lysimeter 2) communication is possible, it was not used in the lysimeter application (and thus not shown in Fig. 4) since each lysimeter was equipped with an SDI-12 soil moisture probe. A disadvantage of the peer-to-peer system for the lysimeter project is that the data from the peer (CR205) to another peer (PC) could not be communicated due to the presence of large buildings between the two sites, which affected the line of sight communication. The major advantage of the master-slave network for the lysimeter project is that it allows for data backup since the data is stored at all the slaves (CR205) as well as the master (CRl0x), which has higher data storage space rather than just the CR205 in the peer-to-peer setup. It can also serve as a signal repeater to transmit the information between the PC and the CR205s for when the distance between them is over one mile. Furthermore, downloading the data is easier with a master-slave network since the PC needs to communicate only to the master rather than connecting to all the CR205 dataloggers.

The program developed in this 
study for the CR205 datalogger enables both types of communication networks. The program has three main functions: 1) obtain data from various sensors; 2 ) store data in table format; and 3) communicate data in wireless mode. The 32000 data points storage capacity of the CR205 allows it to store 15-min-interval lysimeter measurements for over 2 weeks. The user will have 2 weeks to fix any wireless communication problems that may arise before data is lost.

When the Internet network server is in operation, the PC can wirelessly communicate with the CRIOX and all the CR205s via the NLI00 with RF400 radio frequency modem to collect the data (Fig. 3), upgrade the control programs, check the datalogger clock, and display the data at the PC in real time. In case the Internet network server is not available or not in operation, the PC can also use the RF400 radio frequency modem to directly establish wireless communication in the field with the CRIOX at the pump station and all the CR205 dataloggers (Figs. 3 and 4).

If one of the seven dataloggers (six CR205 and one CR10X) experiences problems, the system notifies the person in charge of the project through alarms that can be programmed within the LoggerNet software. The alarm function facilitates correcting the instrument malfunction problems quickly when the problem occurs. Overall, the WDAC network for the multiple-lysimeter project facilitates real-time data monitoring, allows for fast detection of problems, and avoids numerous visits to the site to download data and check instrument status, thus preventing data loss and saving time and project personnel costs.

WDAC PERFORMANCE. The use of WDAC has resulted in the ability to: 1) read and monitor drip and seepage irrigation inflow, drainage outflow, runoff, and soil moisture at four different soil depths in the six lysimeters in real-time from the research office; 2 ) visualize the changes in hydrologic parameters in tabular or graphical formats in real-time; and 3 ) identify problems associated with the functioning of instruments, especially the problems associated with soil moisture sensors, in a timely manner.

Real time soil moisture data has been used in this project to adjust the irrigation timing and interval, estimate percolation to groundwater, and estimate evapotranspiration of vegetable crops such as watermelon (Jaber et al., 2006; Shukla et al., 2006; Srivastava, 2005).

\section{Applications of WDAC systems in horticultural studies}

A WDAC system installed in a large vegetable or citrus farm could be used for effective irrigation management. The WDAC system allows for remote monitoring of soil moisture, irrigation volume, operation of irrigation pumps, and control of irrigation systems from the management office. Soil moisture-based irrigation using real-time data would increase water conservation and reduce nutrient leaching, while optimizing crop production. The number of wireless soil moisture monitoring locations will depend on the number of irrigation zones within a farm, variability in soil physical characteristics, and economic factors. Consider as an example a vegetable farm ( area $=200$ acres $)$ with two main soil types (both sandy soils; soil A covers 50 acres and soil B covers 150 acres) and four irrigation zones of 50 acres each. Assuming that soil $\mathrm{A}$ is contained mostly within an irrigation zone, depending on the cost limitations, two (one each within the two soils) to four (one probe per irrigation zone) soil moisture probes will help better manage the irrigation for the entire farm. It should be noted that there can be considerable variability in soil moisture data obtained from the probes depending on the soil properties and location of the probe. Due to cost considerations, use of several probes within each irrigation zone and soil type may not be possible since some of the more advanced devices cost more than $\$ 1500$ per probe. Some low cost alternatives for capacitance probes exist, however, efforts should be made to ensure that the probe is calibrated for the specific soil type before using it. Use of a non-calibrated probe, even if cheaper, can result in considerable errors in measuring the soil moisture data and result in improper irrigation scheduling. Errors in soil moisture measurements can vary from 13\% to $28 \%$ or higher (C. Pandey and S. Shukla, unpublished data). Therefore, the probe should be selected based on its applicability to the specific soil type and accuracy rather than cost alone.

The WDAC system would also allow for timely troubleshooting (leaks, pump malfunctions, etc.) of the irrigation system based on flow meter readings in different locations in the pipe network. Such tasks could be performed with little manpower. Other parameters not discussed in this study could also be monitored and controlled using WDAC systems. Monitoring and controlling fertigation pumps would enable agricultural operators to ensure timely and accurate delivery of nutrients to horticultural crops.

A WDAC system can also be used as an addition to existing cable-wired datalogging and control systems. This addition would allow the monitoring of additional parameters (e.g., soil moisture) in remote locations through the original datalogging system. This can be achieved by installing the remote sensors with CR2 25 units that can transmit the data to a radio unit installed at the central datalogging system.

\section{Cost comparisons for wireless and manual systems}

Use of wireless technology can greatly reduce monitoring costs. The cost reduction is illustrated for the above-mentioned lysimeter study. The cost (2004 prices) of the monitoring with and without the wireless system for the lysimeter project is presented in Table 2. A part-time personnel (e.g., a graduate student) can remotely monitor the real-time data to determine the schedule of the irrigation practice wherever the Internet is accessible, for example, at the office at UF/IFAS-Immokalee, or at the main campus in Gainesville $(605 \mathrm{~km}$ from the research center). Although irrigation can be automated based on the soil moisture data, due to danger of under or over irrigation (flooding) in case of malfunctioning of controllers, pumps, or electrical switches, the irrigation system was not automated in the lysimeter study.

Real-time monitoring can help avoid excessive watering of crops. In addition, the operational condition of the wireless system can be swiftly recognized by the real-time data monitoring, which helps in alerting personnel to perform repairs soon after data transmission is interrupted.

The traditional manual system for this project would have consisted of two CRI0Xs, each hard wired to a multiplexer for the six lysimeters. The lysimeters were installed in two sets. 
The first consisting of two lysimeters while the other consisted of four lysimeters. The two sets were located at opposite sides of the field. A full-time technician would be needed to visit the study area daily to manage the data collection and analyze the data to decide when to irrigate. During irrigation, the technician needs to visit each lysimeter and spend more than 15 min per lysimeter to download and analyze the data to avoid excessively watering the crops. Daily irrigation can be better managed by using the real time data rather than analyzing the data after irrigation to fine-tune the next day's irrigation. In the event of rainfall, the soil moisture data has to be downloaded to adjust the daily irrigation and to decide about the drainage. In addition, the operational condition of the manual system cannot be recognized until monitoring personnel collects the stored data.

In Florida, the mean annual lightning flash density is 12 flashes/ $\mathrm{km}^{2}$ (Orville and Huffines, 2001). Therefore, lightning damage potential for the instruments for the lysimeter project is very high. The wireless system designed in this study can limit the instrument damage caused by lightning. In the manual system, all the equipments for four lysimeters would have been connected to a CRIOX via a multiplexer. Any lightning strike on either the lysimeters or the data logger will result in damage to all the monitoring systems (CRl0X, multiplexer, and the soil moisture probes) for the four lysimeters. In the WDAC system, each lysimeter has an independent data logger (CR205) wired to the equipment in the lysimeter. Due to the wireless connection with either the central station (peer-to-peer) or the CRIOX (master-slave), it is expected that a lightning strike will damage the monitoring system only in the lysimeter that was directly hit by lightning. A cost comparison was performed between the two systems for a 3-year project (Table 2). A subtotal is shown for the difference in price if there were no lightning strikes during this period, and a total is shown in case the system is hit by lightning once in the 3 years. The no lightning strike subtotal represents the cost of the monitoring systems for areas outside Florida that are less susceptible to lightning. Table 2 shows that the cost of the WDAC system is less than the traditional system for areas with or without lightning damage potential. Moreover, it is clear from Table 2 that more direct hits by lightning will further increase the difference in total cost between the two systems.

The cost of the manual system for the lysimeter project is about twice of the wireless system mainly due to the labor cost and costs associated with replacing damaged instruments and dataloggers (Table 2). In addition, the wireless system offers intangible benefits such as convenient data collection, timely troubleshooting, improved decision making for irrigation and drainage, and less data loss than the manual system.

\section{Summary and conclusions}

The increased need for continuous real-time hydraulic/hydrologic data in large agricultural operations have made the use of wireless technology essential for agricultural water management. There are very few studies that describe agricultural projects using WDAC systems. The essential components and operations of a WDAC system (used for a multiple-lysimeters study) were described in this study. The cost of the manual and the WDAC systems were compared. The example presented in this study could be easily extended to a commercial vegetable/ citrus operation. The advantages of the wireless datalogging network for the lysimeters project include: 1) standardizing the equipment purchase, 2) reducing equipment loss through environmental factors (e.g., lightning strike), 3) improving equipment and project maintenance, 4 ) reducing data loss through faulty equipment, and 5) increasing project personnel efficiency and real-time involvement by a dispersed research team.

\section{Literature cited}

Campbell Scientific, Inc. 2002a. NL100/ 105 Network link interface instruction manual. Campbell Scientific, Inc., Logan, Utah.

Campbell Scientific, Inc. 2002b. Loggernet user's manual, Version 2.0. Campbell Scientific, Inc., Logan, Utah.

Campbell Scientific, Inc. 2003. CR200 datalogger and CR2 25, CR210, or CR215

Table 2. Cost comparisons between a manual data collection and control system and a wireless data acquisition and control system (WDAC) for a 3-year six-lysimeter project for evapotranspiration and water quality studies.

\begin{tabular}{|c|c|c|c|c|}
\hline \multirow[b]{2}{*}{ Item } & \multicolumn{2}{|l|}{ Wireless } & \multicolumn{2}{|l|}{ Manual } \\
\hline & Quantity/description & Costs $(\$)$ & Quantity/description & Costs $(\$)$ \\
\hline \multirow[t]{5}{*}{ Instruments } & six CR205s with radios & 3,420 & two CRI0X & 2,320 \\
\hline & one NL100 & 450 & six Sentek EasyAG probes & 4,860 \\
\hline & two RF400 radios & 880 & 24 flow meters & 7,200 \\
\hline & one $6 \mathrm{dBd}$ Yagi antenna & 200 & & \\
\hline & 24 flow meters & 7,200 & & \\
\hline Human resources & one part-time technician for 3 years & 45,000 & one full-time technician for 3 years & 105,000 \\
\hline \multicolumn{2}{|c|}{ Subtotal cost (without lightning) } & 63,170 & & 120,580 \\
\hline \multirow[t]{2}{*}{ Lightning damage $^{\mathrm{z}}$} & one CR205 with radio & 570 & one CRI0X & 1,160 \\
\hline & one Sentek EasyAG probe & 810 & one multiplexer & 600 \\
\hline
\end{tabular}

${ }^{2}$ Lightning is assumed to directly hit the set of four lysimeters located at one end of the field. Two other lysimeters located at the other end of the field will not be affected. 
datalogger with spread spectrum radio operator's manual. Campbell Scientific, Inc., Logan, Utah.

Campbell Scientific, Inc. 2004. CRI0X measurement and control system. Campbell Scientific, Inc., Logan, Utah.

Campbell Scientific, Inc. 2005. Instructional manual for RF 400/RF415 spread spectrum data radio/manual. Campbell Scientific, Inc., Logan, Utah.

Cheek, S. and R. Wilkes. 1994. Monitoring processes using wireless data acquisition. Water Eng. Mgt. 144(9):22-23.

Cockerham, S.T. and T.R. Ortega. 1989. Remote data acquisition for agricultural experiment station management. Appl. Agr. Res. 4(2):144-147.

Damas, M., A.M. Prados, F. Gomez, and G. Olivares. 2001. HidroBus system: Fieldbus for integrated management of extensive areas of irrigated land. Microprocessors Microsystems 25:177-184.

Delin, K.A., S.P. Jackson, D.W. Johnson, S.C. Burleigh, R.R. Woodrow, J.M. McAuley, J.M. Dohm, F. Ip, T.P.A. Ferré, D.F. Rucker, and V.R. Baker. 2005. Environmental studies with the Sensor Web: Principles and practice. Sensors 5:103-117.

FTS Forest Technology Ltd. 2000. SDI12. 30 Jan. 2006. <http://www.ftsinc. com/Features/About_SDI12.htm>.

Glasgow, H.B., J.M. Burkholder, R.E. Reed, A.J. Lewitus, and J.E. Kleinman. 2004. Real-time remote monitoring of water quality: A review of current applications, and advancements in sensor, telemetry, and computing technologies. J. Expt. Marine Biol. Ecol. 300:409-448.

Jaber, F.H., S. Shukla, and S. Srivastava. 2006. Recharge, upflux and water table response for shallow water table conditions in southwest Florida. Hydrological Processes 20(9):1895-1907.

Lundquist, J.D., D.R. Cayan, and M.D. Dettinger. 2003. Meteorology and hydrology in Yosemite National Park: A sensor network application, p. 518-528. In: F. Zhao and L. Guibas (eds.). Information processing in sensor networks,. SpringerVerlag, Heidelberg, Germany.

Maes, M.E. and J.R. Steffey. 1996. New techniques provide solutions to data communication dilemmas in water districts. Water Eng. Mgt. 143(7):22-25.

Microsoft. 1999. Microsoft Windows 2000 Professional. Microsoft Corp., Redmond, Wash.

Orville, R.E. and G.R. Huffines. 2001. Cloud-to-ground lightning in the United States: NLDN results in the first decade, 1898-98. Monthly Weather Rev. 129(5):1179-1193.

Reed Business Information. 2003. Remote monitoring, logging and control. 30 Jan. 2006. <http://www.ferret.com. $\mathrm{au} /$ articles $/ 4 \mathrm{c} / 0 \mathrm{c} 01944 \mathrm{c}$.asp $>$.

Sauter, K.A. and J.J. McDonnell. 1994. Automated system for measuring snow surface energy balance components in mountainous terrain. Hydrological Processes 8(5):437-446.

Sentek Pty Ltd. 2002. EnviroSMART ${ }^{\mathrm{TM}}$ \& EasyAG ${ }^{\mathrm{TM}}$ SDI-1 2 probe interface manual. Sentek Pty. Ltd., Stepney, Australia.

Shukla, S. and C. Pandey. 2003. Development and evaluation of vegetable irrigation management practices for water use and quality in southwest Florida. Interim Rpt. No. WRP-0003. Florida Fruit Veg. Assn., Orlando.

Shukla, S., S. Srivastava, and J.D. Hardin. 2006. Design, construction, and installation of large drainage lysimeters for water quantity and quality studies. Appl. Eng. Agr. 22(4):529-540.

Southeast Regional Climate Center. 2005. Immokalee 3NW, Florida-Climate summary. I Feb. 2006. <http://cirrus. dnr.state.sc.us/cgi-bin/sercc/cliMAIN. pl?fl4210>.

Srivastava, S. 2005. Crop coefficient and water quality for watermelon and bell pepper under drip and seepage irrigation. Masters Thesis, Univ. of Florida, Gainesville.

Teillet, P.M., R.P. Gauthier, A. Chichagov, and G. Fedosejevs. 2002. Towards integrated Earth sensing: Advanced technologies for in situ sensing in the context of Earth observation. Can. J. Remote Sensing 28:713-718. 